\title{
The Atomki Accelerator Centre
}

\author{
S. Biri ${ }^{\circledR}$, I. K. Vajda, P. Hajdu, R. Rácz, A. Csík, Z. Kormány, Z. Perduk, F. Kocsis, \\ I. Rajta
}

Institute for Nuclear Research (Atomki), Bem tér 18/c, Debrecen 4026, Hungary

Received: 5 November 2020 / Accepted: 11 February 2021

(C) The Author(s) 2021

\begin{abstract}
In this paper, the Atomki Accelerator Centre (AAC, Debrecen, Hungary) incorporating several small-sized particle accelerators is reviewed. The energy range of our accelerators for proton beam is between $50 \mathrm{eV}$ and $20 \mathrm{MeV}$. The technical and personnel organization of AAC is presented together with the rules of beamtime requests and usage. Three of our accelerators (Cyclotron, ECRIS, Tandetron) are described in detail with their technical descriptions and with the main application fields. As an example for highlights, a series of unique low-energy ion-sample irradiations and post-treatments are shown which, by our hopes and plans, form a bridge between physics and biology.
\end{abstract}

\section{Introduction}

\subsection{History}

The Institute for Nuclear Research (Atomki, Debrecen, Hungary) [1] was founded in 1954. Throughout its 66 years history, significant part of scientific research and the published results were achieved based on our particle accelerators. The development of accelerators started in the late 60s with the designing and building of two Van de Graaff accelerators. In the subsequent decades, the developments continued, but gradually the later accelerators were already purchased from professional factories.

Between 1970 and 2009, the accelerators belonged to that departments which developed and/or used the given accelerator. In order to reinforce and concentrate the infrastructure backbone of Atomki, a new division was established in 2009: the Atomki Accelerator Centre (AAC) [2]. The new division has numerous advantages. The staff of AAC (physicists, engineers and operators, cca 15 persons) can be re-distributed or even concentrated to a given task or to a given accelerator. The operators can work at more than one accelerator, they can help and teach each other. The maintenance of the accelerators can be organized optimally, the necessary operational and spare tools (vacuum technics, power supplies, radiation safety, etc.) are always available somewhere within the AAC. The distribution of the beamtime among different accelerators is much more optimal and equal. Technically, the accelerators offer the possibility of selecting ions with various charge states, energies and beam intensities. By today, the AAC became a strong and permanent organizational unit of Atomki, it is well

\footnotetext{
a e-mail: biri@ atomki.hu (corresponding author)
} 
known for our present and future partners. The AAC got into several domestic and European scientific databases.

\subsection{Beams and beamtimes}

Table 1 summarizes the main features of our main past and present ion accelerators. The proton energy ranges are in the 6th column. The maximal kinetic energy for other particles is the product of the charge and the achievable high voltage. For example, the highest charge state the ECR ion has delivered so far is 30 (gold beam). Setting the highest available platform (accelerating) potential to $30 \mathrm{kV}$ than the total kinetic energy of this beam is $900 \mathrm{keV}$. More technical details on the accelerators are in [3] and in [4].

The distribution and supplying of beamtime are carried out by strict rules. Twice a year (usually in December and in June), the users may apply for beamtime for the next halfyear. Users can be our researchers (internal users) or external ones, too. In the past 10 years, the ratio of external users (domestic and foreign together) was around $30 \%$. In this way, our accelerators are open-access devices. An application for beamtime can be done by filling out an on-line data-sheet in the homepage of the institute [5]. In the same place, there is a detailed Beam Request Rule documentation containing detailed description of the accelerators and the cost of beamtime. The Program Advisory Committee (PAC) judges the requests twice a year and makes a proposal for the director. The director comes to the final decision on the allowed beamtimes (hours) and on the price of one hour for each requesting user. This decision is sent to the users and also published in the intranet. Then, the users can allocate the necessary/allowed days for their research by an on-line system which is part of our corresponding software (Zimbra). The allocation is confirmed by the chief engineer of the given accelerator. The chief engineers of the two largest accelerators (Cyclotron and Tandetron) are in continuous contact in order to distribute the beamtime and the staff optimally and equally throughout the half-year, especially pay attention to the long, continuous irradiations (usually from Monday morning till Friday evening) which needs the optimal day-and-night distribution of the operator staff. The daily and monthly beamtime usage can be also followed on the homepage of AAC. In Fig. 1, the delivered beamtime hours are shown in recent years. The Tandetron started its operation in 2015. The last year of the VdG-5 was 2019. In 2018, the building of the full beamline system limited the beamtime at the Tandetron.

\subsection{Accelerator Centre}

Exams are regularly organized for the operator staff. Each operator has to pass exams in the following subjects: vacuum theory, vacuum practice, radiation safety, labor safety. Then, other exams for the given accelerator are required: accelerator theory (basics, principles), accelerator operation. The interest of the exam board (whose members are usually the AAC Head and two accelerator engineers) is to teach and to positively motivate the operators. That is why, in case of an unsatisfactory result, a second exam is required and hold within a couple of weeks. Presently, all of the operators can operate one accelerator professionally (Cyclotron or Tandetron) and some of them can serve as assisting operator at another accelerator.

The $1 \mathrm{MeV}$ VdG-1 accelerator (see Table 1) was built as a test-bench before designing and building the $5 \mathrm{MeV}$ VdG-5. Surprisingly, this small test-accelerator is still operational and in the subsequent years its high-voltage system will be upgraded. The $5 \mathrm{MeV}$ VdG-5 accelerator served excellently between 1971 and 2019. In 2019, its technical condition went down (and even the building incorporating the accelerator was in a bad state) and by the appearance of 


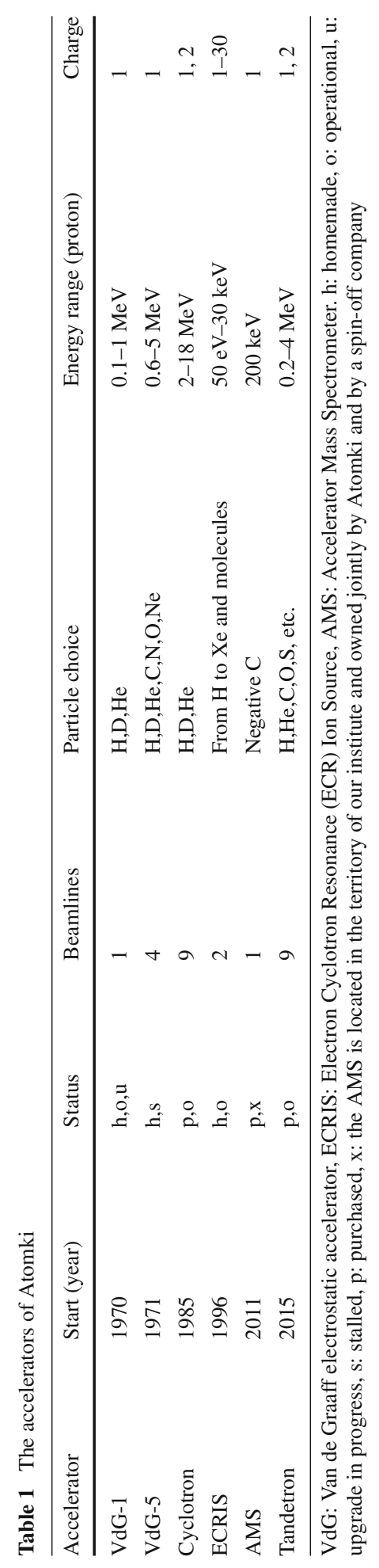




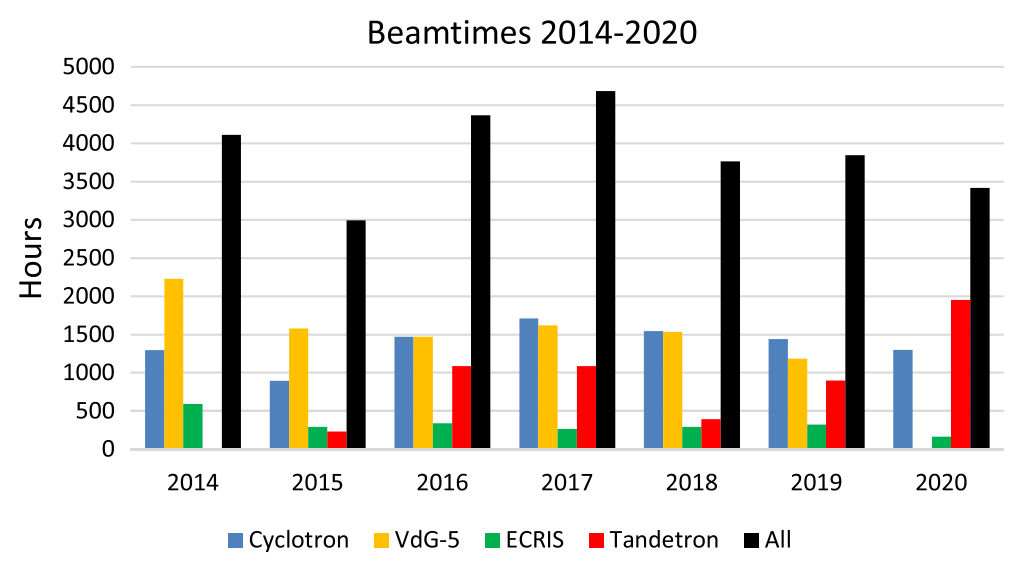

Fig. 1 Delivered beamtimes (hours) by some of the Atomki accelerators in recent years

the Tandetron the users gradually moved from the VdG-5 to the Tandetron. Finally, at the beginning of 2020 we made the decision to stop VdG-5. Some technical details of the VdG-1 and VdG-5 accelerators can be found in the AAC homepage [2]. The fix-voltage (200 kV) AMS accelerator [6] is located in the territory of our institute, owned jointly by Atomki and by a spin-off company and operated by the latter one.

In the subsequent sections, our presently operating accelerators are shown in detail: the MGC-20E Cyclotron, the homemade ECR Ion Source and the HVEE-made Tandetron. In the highlights section, a series of non-conventional experiments are shown at the ECR ion source.

\section{Cyclotron}

The MGC-20E Cyclotron of the center was manufactured by the Efremov Institute, Russia and has been in service since 1985. It is a compact isochronous cyclotron being capable of accelerating the four lightest ions, limited mainly by its internal low-power ion source. The available beam types, energies and intensities are summarized in Table 2.

Table 2 Available cyclotron beams with maximally extracted beam intensities and the required harmonic mode of operation of the RF-system

\begin{tabular}{llll}
\hline Particle & Energy $[\mathrm{MeV}]$ & RF harmonic mode & Max. extracted current $[\mu \mathrm{A}]$ \\
\hline $\mathrm{p}$ & $2-2.6$ & 3 & 20 \\
& $2.6-18$ & 1 & 50 \\
$\mathrm{~d}$ & $2.3-5.2$ & 3 & 20 \\
& $5.2-10$ & 1 & 50 \\
${ }^{3} \mathrm{He}^{2+}$ & $4-8.0$ & 3 & 2 \\
& $8.0-27$ & 1 & 8 \\
${ }^{4} \mathrm{He}^{2+}$ & $3.5-10.4$ & 3 & 5 \\
& $10.4-20$ & 1 & 20 \\
\hline
\end{tabular}




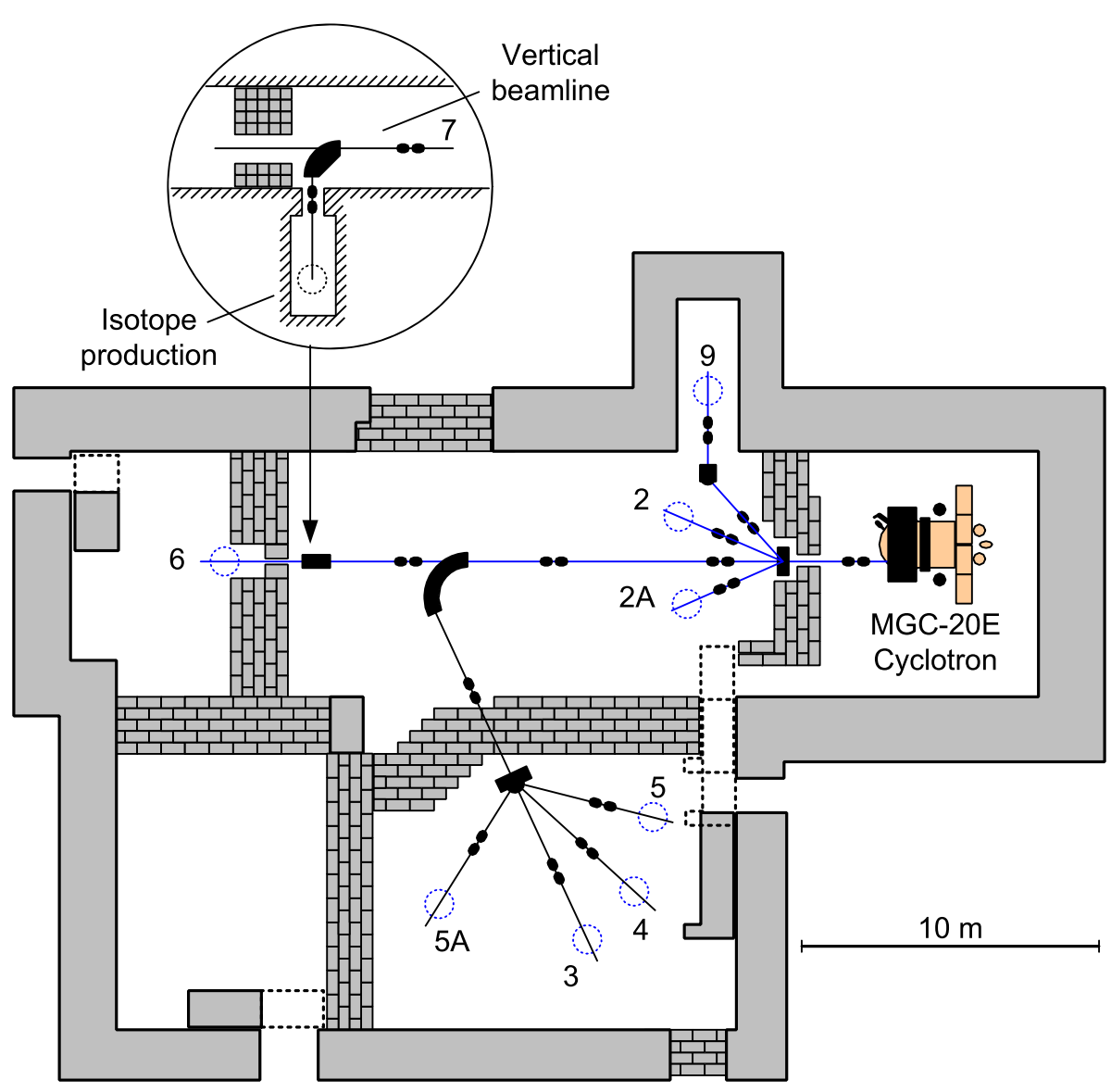

Fig. 2 Cyclotron vaults and beamlines

The beam transport system of the cyclotron can deliver beams into 4 different target vaults with a total number of 9 target locations. The layout of the beam transport system is given in Fig. 2. The unique vertical beamline to the target in the basement (No. 7) is dedicated for radioisotope production with high beam power. The transport system is also equipped with an analyzing magnet (for targets No. 3, 4, 5 and 5A) which can decrease the inherent energy spread of the cyclotron beam to the level required by nuclear physics research programs.

The major subsystems of the cyclotron have been renewed and upgraded systematically. First, the vacuum and control systems were renewed in the end of the 90s. The manual control system was replaced by programmable logic controllers (PLC) providing computer support for the operation. Later, the obsolete analog electronics in the NMR field stabilization system of the analyzing magnet was replaced by a novel home-made digital unit. Lastly, a new $2 \times 2.4$ $\mathrm{kW}$ solid-state broadband RF driver amplifier was built and installed, replacing the original tube-based amplifier. Since the production of the proprietary RF-tubes has been discontinued by the manufacturer, this development was crucial to assure the long-term operation of the machine.

The cyclotron has a broad range of utilization extending from basic research to industrial and medical applications. Major fields are: 
- Nuclear reactions of astrophysical relevance are studied using the activation method. Cross sections of many reactions relevant for the astrophysical p-process as well as of the ${ }^{3} \mathrm{He}(\alpha, \gamma){ }^{7} \mathrm{Be}$ reaction have been measured [7], which contributed to the better understanding of the investigated stellar processes (Target No. 9).

- Super- and hyper-deformed rotational bands of several actinide nuclei are studied by the split-pole magnetic spectrometer and the unique detectors built in Atomki [8]. The hyper-deformed rotation bands of the ${ }^{236} \mathrm{U}$ have been identified for the first time (Targets No. 3, 4 and 5A).

- Thin Layer Activation of different machine parts and material samples is performed on external beam. Stacked foils are irradiated to determine excitation functions and yields of different charged particle-induced nuclear reactions and radioisotopes are produced as tracers in different processes (Target No. 2A).

- Neutron physics, dosimetry research and calibration of neutron detectors are done with quasi mono-energetic neutrons. For radiobiological and radiation hardness studies [9], high-intensity fast neutrons of continuous energy distribution are used (Targets No. 2, 5).

- Radioisotopes are produced by irradiation and separated by radiochemical methods for nuclear medicine in PET imaging and/or for targeted radionuclide therapy in oncology. The home-made mini-PET camera with ${ }^{52} \mathrm{Mn}$ and ${ }^{64} \mathrm{Cu}$ radioisotopes are used for phenotyping of agricultural crops [10]. Some strong gamma emitter radioisotopes are also used in nuclear industry to monitor the nuclear power plant for leaking such as ${ }^{48} \mathrm{~V}$ and ${ }^{56}$ Co (Targets No. 6, 7).

\section{Electron cyclotron resonance ion source}

The second-generation electron cyclotron resonance (ECR) Ion Source (ECRIS) has been operating in Atomki since 1996. Instead of the usual application of such sources, this "homemade" Atomki-ECRIS [11] does not deliver ion beams for high energy accelerators but provides versatile low-energy ion beams and plasmas for atomic physics research [12], plasma investigations [13] and materials science [14]. A numerical code for plasma simulation is developed by the Atomki group [15] and used by several ECR-groups throughout the world.

The total kinetic beam energy can be varied between $50 \mathrm{eV}$ and $900 \mathrm{keV}$ depending on the extraction voltage and on the charge state of the accelerated ion species. The cross section drawing of the mechanical configuration of the source is presented in Fig. 3. The ion beam is extracted from the plasma by a changeable tip puller electrode and is focused by two electrostatic Einzel lenses.

The plasma (heated by $14 \mathrm{GHz}$ microwave at several hundred watts power) is confined in the cylindrical plasma chamber by B-minimum magnetic field. The B-field is produced by the combined usage of hexapolar permanent magnet and two room-temperature solenoids. The radial magnetic field measured at the plasma chamber wall is about 1.2 Tesla while the axial magnetic peak fields along the axes of the plasma chamber are $1.26 \mathrm{~T}$ at the injection side and $0.95 \mathrm{~T}$ at the extraction side. One of the unique features of our ion source is that the mechanically two solenoids electrically mean three independent coils (since 2018 with an extra pancake between the iron yokes, see Fig. 3). Therefore, it is possible to vary the minimum field (typically $0.39 \mathrm{~T}$ ) independently from the peak fields. In this way, the gradient of the B-field at the resonant zone can be finely tuned. This fine-tuning significantly increased the achievable beam currents, furthermore opens the possibility to investigate kinetic plasma instabilities which are appearing at low gradient values [16] and nowadays is hot-topic in plasma physics. 


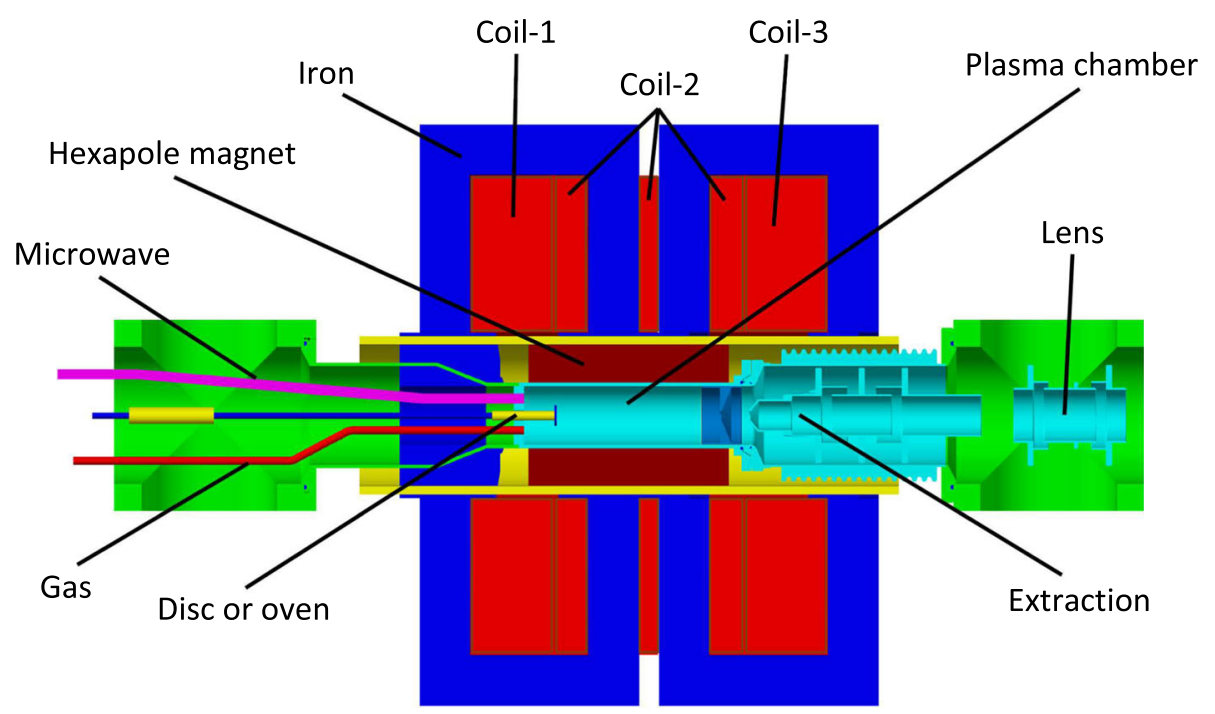

Fig. 3 The cross section drawing of the Atomki-ECRIS

Due to the effective energy transfer from the microwave to the plasma electrons this relatively old ECRIS can produce reasonably high charge state of ions. For example, fully stripped $\mathrm{Ne}$ ions or He-like $\mathrm{Ar}^{16+}$ beams were generated. Proton beams and multiply charged ions of $\mathrm{He}, \mathrm{C}, \mathrm{N}, \mathrm{O}, \mathrm{Ne}, \mathrm{Si}, \mathrm{Ar}, \mathrm{Kr}, \mathrm{Xe}$ are used in daily routine with high intensity. Due to the high ionization efficiency and based on the several vaporizations technics available in Atomki our ECRIS can produce ion beams from a variety of materials, even from solids. Au, Ag, Fe, $\mathrm{Ni}, \mathrm{C}_{60}$ ion beams are provided by applying the MIVOC method [17], oven and sputtering techniques [18]. It was also proved that besides highly charged positive atomic ions, $\mathrm{H}^{-}, \mathrm{O}^{-}$, $\mathrm{OH}^{-}, \mathrm{O}_{2}^{-}, \mathrm{C}^{-}, \mathrm{C}_{60}^{-}$negative ions and $\mathrm{H}_{2}^{+}, \mathrm{H}_{3}^{+}, \mathrm{OH}^{+}, \mathrm{H}_{2} \mathrm{O}^{+}, \mathrm{H}_{3} \mathrm{O}^{+}, \mathrm{O}_{2}^{+}$positive molecular ions can also be generated [19] with appropriate intensities. Negative ion beams of several $\mu \mathrm{A}$ and positive molecular ion beams in the $\mathrm{mA}$ range for low-energy atomic and molecular physics research were obtained. The ion selection of the Atomki-ECRIS can be seen in Figs. 4 and 5 showing the highest beam currents and charge states ever produced from gases and from solids, respectively.

\section{Tandetron}

The new Tandetron accelerator was installed in 2014 by High Voltage Engineering Europa B.V. [20], and was equipped in 2015 with a duoplasmatron ion source (Model 358) as well as a 9-port switching magnet directly at the exit of the machine. In the first 3 years of operation [4], four temporary beamlines were gradually built on the switching magnet: nuclear physics, nuclear astrophysics, external beam, and a newly developed scanning ion nanoprobe setup.

With a subsequent financial support, we have upgraded the setup with two multicusp ion sources (for hydrogen and helium beams, SO120 and SO130, respectively) and with a 90-degree analyzing magnet. The duoplasmatron ion source has been changed by a cesium sputter ion source (Model 860C), that is capable to produce negative ion beams from most of the heavy elements. We have moved the switching magnet from its temporary position to 


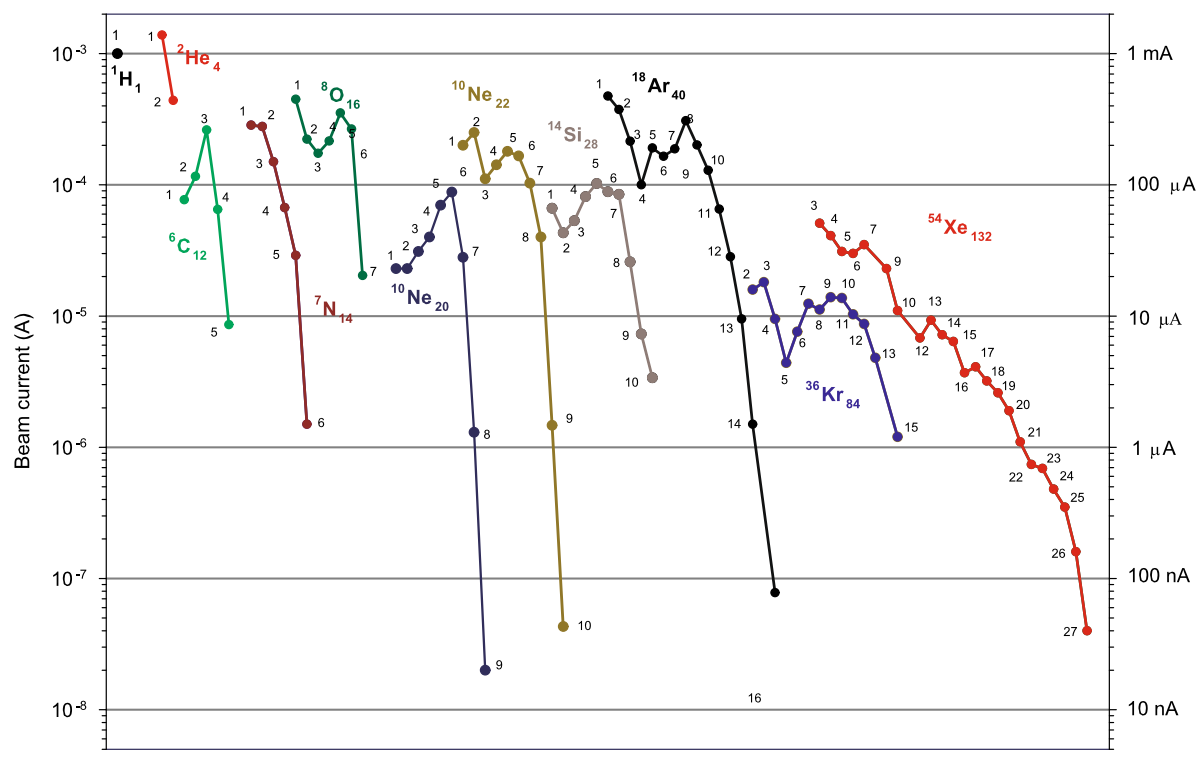

Fig. 4 Beam currents as function of the charge state corresponding to ion species produced from gaseous sources

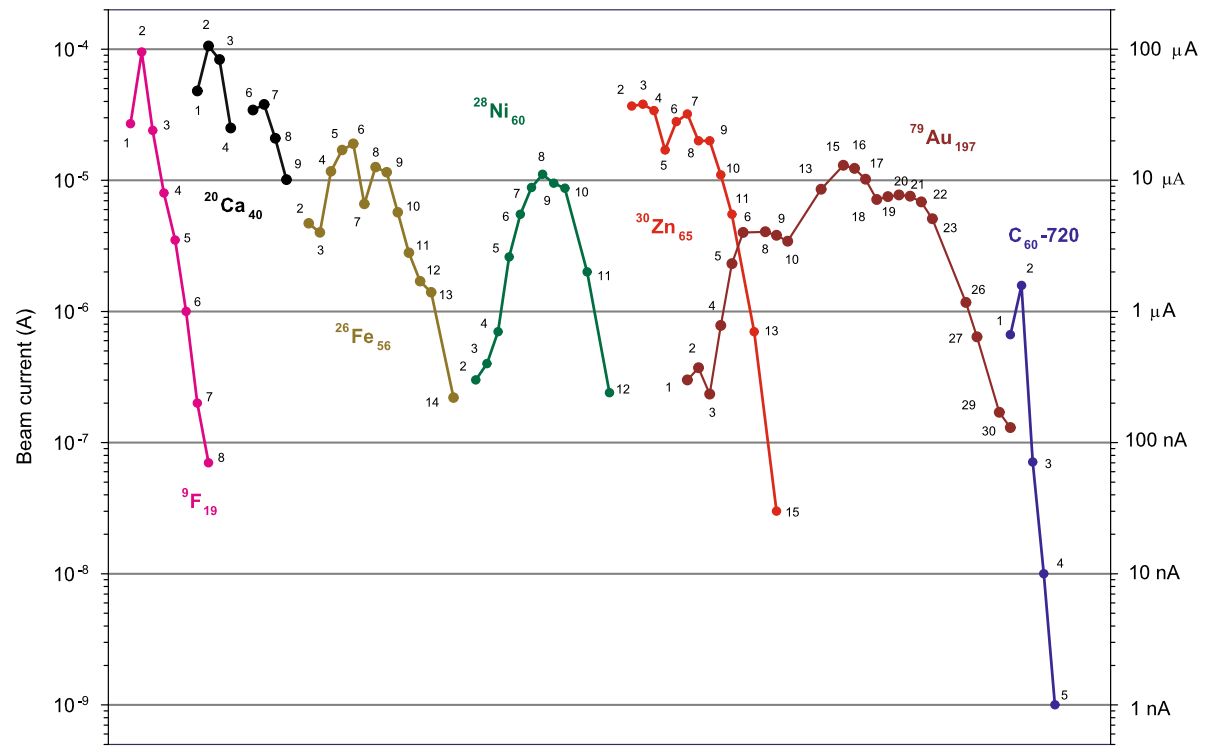

Fig. 5 Beam currents as function of the charge state corresponding to ion species produced from solids

the exit of the analyzing magnet. After that, the 9 final beamlines were designed, built and now gradually are given to the users (see Fig. 6, Phase 2) [21].

- The nanoprobe has been moved to the final position on the right 10-degree line of the switching magnet. 


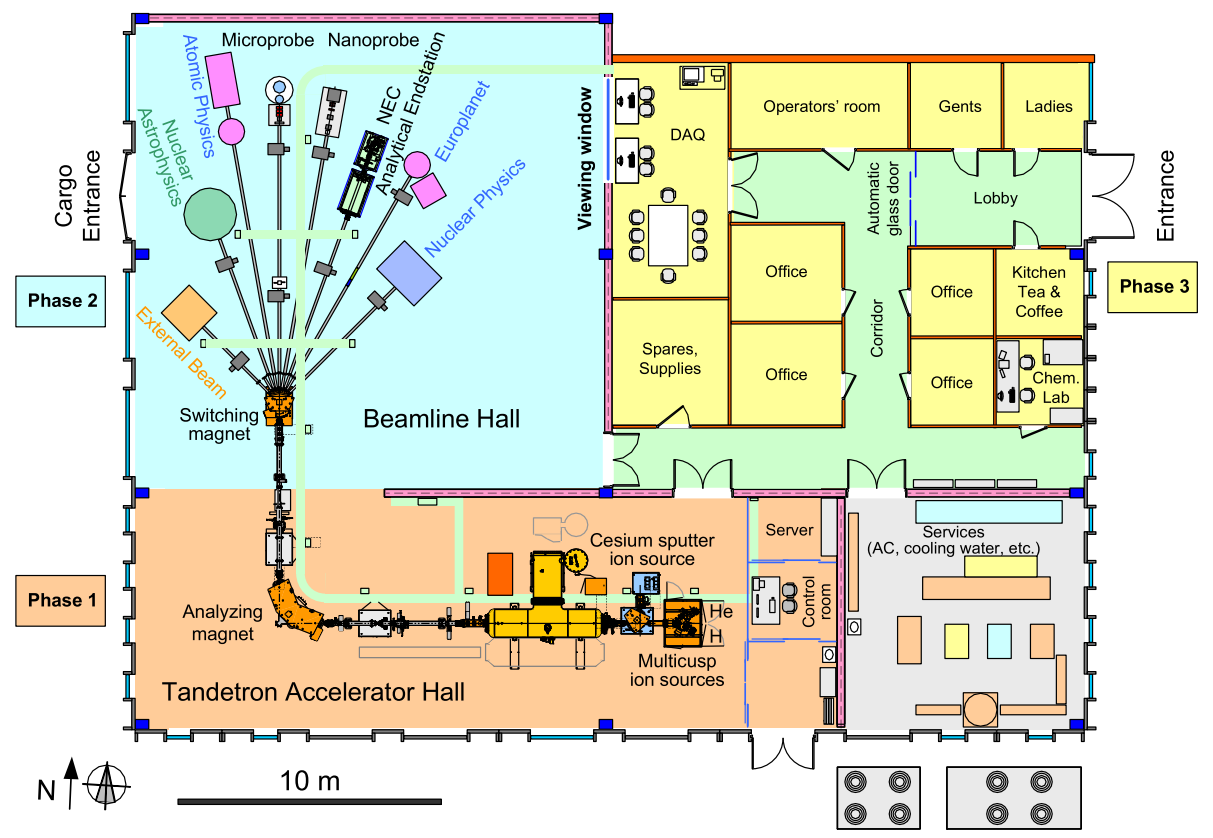

Fig. 6 Tandetron Laboratory layout. Note: Phase 1 and 2 are completed, phase 3 is still in planning stage

- The microprobe was the flagship beamline station of the old $5 \mathrm{MeV}$ Van de Graaff accelerator for over 20 years [22]. Now it is operated on the zero-degree port of the Tandetron allowing much better quality ion beam (stability, beam size, etc.).

- The new RC43 analytical endstation (made by National Electrostatics Corp. [23]) has been installed on the right 20-degree beamline. Many Ion Beam Analytical (IBA) methods will be available here: PIXE (Proton-Induced X-ray Emission), RBS (Rutherford Backscattering Spectrometry), ERDA (Elastic Recoil Detection Analysis), IBIL (Ion Beam-Induced Luminescence) and Channeling. The sample holder is equipped with a 5-axis goniometer, and can be heated up to $500{ }^{\circ} \mathrm{C}$ in situ.

- The external-beam setup was upgraded with a compact X-ray detector cluster featuring three Be windowed and one thin windowed SDD (Silicon Drift Detector) made by RaySpec Ltd [24]. The setup also includes two lasers and an optical microscope for perfect sample positioning.

- In addition to various aspects of IBA, the Tandetron accelerator was planned to serve several users and topics in fundamental research. The high achievable beam intensities combined with good energy resolution and long term stability make the Tandetron accelerator ideal for nuclear astrophysics research. A dedicated beamline for astrophysical experiments has thus been set up, where some key reactions of stellar hydrogen burning processes have already been studied [25,26].

- Ions of different species and energies can mimic the effects of cosmic rays and stellar wind on low temperature $(10-80 \mathrm{~K})$ ice mixtures. The Ice Chamber for Astrophysics/Astrochemistry (ICA) at Atomki, a Transnational Access (TA) facility of the EUROPLANET 2024 RI Project Consortium, is designed to investigate those irradiation effects on analogues of interstellar and Solar System ices. The various beams are produced by the Tandetron accelerator. One of its main advantages is that the multicusp 
and sputtering ion sources can be kept in standby mode simultaneously, and changing ion species or energies takes only a short time (e.g., it takes only one hour from $1 \mathrm{MeV}$ protons to $6 \mathrm{MeV} \mathrm{S}^{2+}$ ions). Various TA research programs have been accepted by the Project Consortium for the next year, the first one is already running. The major goal of the experiments is to study space relevant ices under different ion-impact conditions systematically, to understand the origin of the building blocks of life.

- A high-efficiency electron positron spectrometer was built at Atomki to search for a light particle, and achieved breakthrough results [27,28]. The spectrometer has been moved from the VdG-5 to the Tandetron accelerator and refurbished with the help of state-ofthe-art detectors and data acquisition system. It is now used there to learn more about the properties of the famous new particle (called X17) introduced at Atomki [29].

As written above, most of the exit ports of the 9-branch switching magnet are already occupied and used in daily/weakly routine. In principle, all beamlines (including the presently not-used ones, as well) will be available for present and future users, both internal and external.

\section{Highlight}

\subsection{The Atomki-ECRIS as direct implanter}

It is well known that most ECR Ion Sources in the world are used as primary ion beam injectors for post-accelerators operating mainly for particle and nuclear physics. In Atomki however, our ECRIS operates as an independent, low-energy accelerator. One of its missions is to produce low-energy ion beams for modification and functionalization of solid surfaces, especially surfaces of medical implants and restorations. The basic question always is: what sample material should be irradiated by what particles for what purposes? There are several known material "pairings" (targets-projectiles) such as zirconium-silicon in the case of prostheses; and titanium-gold, titanium-silver and titanium-calcium for implants, which are important for, e.g., dentists. Surface modification in other labs usually requires a special equipment which delivers one certain type non-conventional ion beam at appropriate energy and dose, required for these irradiations. As another solution, during the last decade we formed our ECR Ion Source into a multipurpose low-energy implanter for surface modifications in wide range of projectile type, charge, energy and dose. In Sect. 3, the available particles with electrical beam intensities were shown. In terms of irradiation dose the ECRIS of Atomki is able to provide about $10^{17}$ ions $/ \mathrm{cm}^{2}$ within reasonable irradiation time (hours) in case of low and middle-charged beams, especially from gases. We are also able to produce plasmas from solid materials using various techniques. These techniques have been implemented for many years partly from other groups, partly by us.

\subsection{Collaboration between physicists and medical doctors}

In order to use our instruments and knowledge in physics for medical purposes, our institute has been collaborating with the Department of Biomaterials and Prosthetic Dentistry of the University of Debrecen for a couple of years. We work together on several joint research topics, all of them aiming the surface modification of dental restorations and implants by low-energy ion beams. The implanting depth is usually in the upper 5-200 nm layer of the material surface. The irradiation of the samples is followed by cell-growing on the modified surface or by bonding strength investigations. The aim is the "functionalization" of the medical sample for a well-developed goal, e.g., to improve the bonding strength between the 
metal and living cells, or increase the lifespan or the bacterial properties, etc. Recently, we irradiated zirconia and titanium-oxide target materials with silicon, silver, gold and calcium ion beams provided by the Atomki-ECRIS.

Below, we report some details on the most promising irradiations, as examples.

1. Silicon ions into $\mathrm{ZrO}_{2}$. In dentistry, it is necessary to increase the bonding strength between various bioinert zirconia ceramics and the luting cement in order to extend the lifetime of the restoration. Our ion source was used to implant Si ions just under the surface of the $\mathrm{ZrO}_{2}$ on nanoscale to improve chemical bonding in between the bonding agent and the zirconium-dioxide. The stronger a material, the higher the load at brake value. We were able to implant $\mathrm{Si}$ ions in a controlled way into the upper few 10 nanometers on the surface of zirconium ceramics through a fine metal mesh. The SRIM simulation and the Secondary Neutral Mass Spectrometry (SNMS) measurement results were in good agreement as shown in Fig. 7. We found that the load at brake is higher when we apply the combination of $3 \mathrm{kV}$ extraction ECRIS voltage, post-irradiated by oxygen ions and even venting the vacuum chamber with oxygen [30].

2. Calcium and silicon ions in titanium. Similar to irradiation into zirconium, significant changes were achieved on the surface of titanium by these irradiations. We modified the surface of titanium not only with silicon ions but also with calcium ions. As a result of treatments, the viability of the cells planted on titanium was increased [31].

3. Silver ions in titanium. The antibacterial effect of silver is well known in medicine. The Ti samples in some experiments were just irradiated by Ag ions, in some other cases were coated by Ag-evaporation (PVD) and in an experiment were treated by both methods. We demonstrated that the formed silver nanoparticles are not cytotoxic or at least does not decrease significantly the cell viability [32] and are still antibacterial. It is very important that, these nanoparticles should not be released into human tissue. The best mechanical bond between the nanoparticle and the titanium is reached by irradiating the titanium

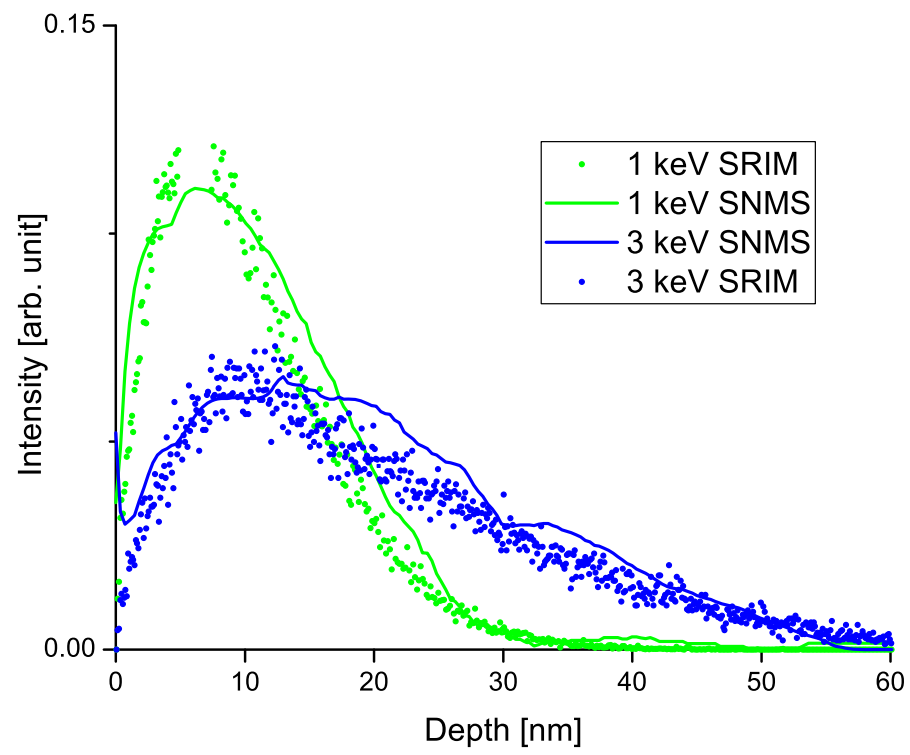

Fig. 7 Comparison of the depth distributions of $1 \mathrm{keV}$ (green curve and dots) and $3 \mathrm{keV}$ (blue curve and dots) silicon atoms calculated by SRIM and measured by SNMS 

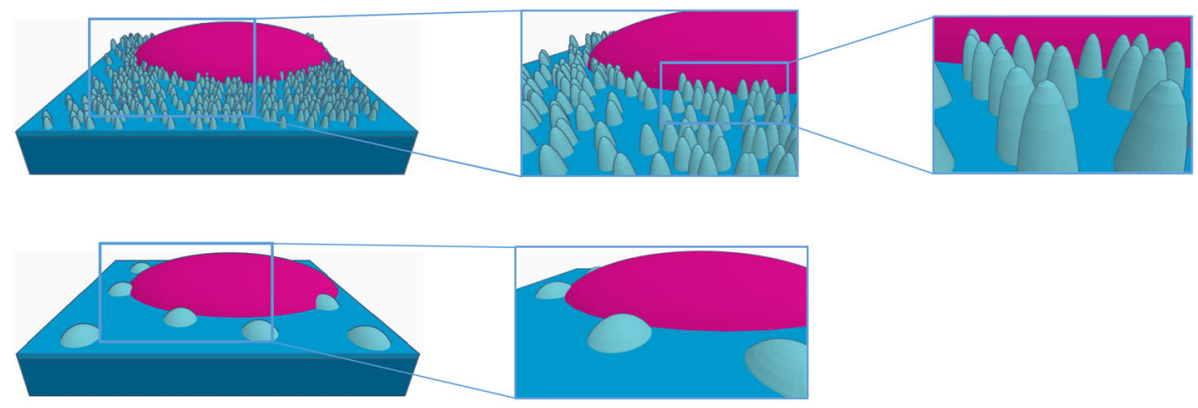

Fig. 8 Schematic of $60 \mathrm{~nm}$ (upper) and $368 \mathrm{~nm}$ (bottom) average diameter Ag nanoparticles formed on the titanium surface, on which cell culture was applied

surface with Ag ions (ECR beam) before creating the Ag nanoparticles (PVD+heating) on it, as Fig. 8 shows [14].

These results indicate that the nature cannot be considered as independent, "parallel" studies, but it is more similar to a ball where you are getting closer and closer to another field as you get more and more deeply in that ball. We definitely continue both our ECRIS beam developments and the application of these beams to medical purposes.

\section{Summary}

In the paper, the particle accelerators of Atomki were reviewed. All of our accelerators belong to "small-sized" facilities, but their wide energy range made possible to carry out a large number of various ion-target experiments in many fields for decades and will in the future, as well. Recently, the most prospective research fields at AAC are: nuclear physics, nuclear astrophysics, laboratory astrochemistry, ion beam applications and medical applications.

Acknowledgements The financial support of the Hungarian Academy of Sciences (Infrastructure grants), the Hungarian Government, Economic Development and Innovation Operational Program (GINOP-2.3.3-152016-00005 and GINOP-2.3.3-15-2016-00034) grants, co-funded by the EU, is acknowledged. The RADIATE project under the Grant Agreement 824096 from the EU Research and Innovation program HORIZON 2020 is also acknowledged. Financial support of the Hungarian NKFI Foundation (K124810) is acknowledged. Scanning electron microscopy measurements were taken with the support of the GINOP-2.3.3-15-2016-00029 project.

Funding Open access funding provided by ELKH Institute for Nuclear Research.

Open Access This article is licensed under a Creative Commons Attribution 4.0 International License, which permits use, sharing, adaptation, distribution and reproduction in any medium or format, as long as you give appropriate credit to the original author(s) and the source, provide a link to the Creative Commons licence, and indicate if changes were made. The images or other third party material in this article are included in the article's Creative Commons licence, unless indicated otherwise in a credit line to the material. If material is not included in the article's Creative Commons licence and your intended use is not permitted by statutory regulation or exceeds the permitted use, you will need to obtain permission directly from the copyright holder. To view a copy of this licence, visit http://creativecommons.org/licenses/by/4.0/. 


\section{References}

1. www.atomki.hu/en

2. www.atomki.hu/en/departments/1/introduction

3. I. Vajda et al., AIP Conf. Proc. 1852, 060002 (2017). https://doi.org/10.1063/1.4984866

4. I. Rajta et al., Nucl. Inst. Meth. A 880, 125 (2018). https://doi.org/10.1016/j.nima.2017.10.073

5. www.atomki.hu/en/pac

6. www.atomki.hu/en/topics/view/36

7. T. Szücs et al., Phys. Rev. C 99, 055804 (2019). https://doi.org/10.1103/PhysRevC.99.055804

8. A.J. Krasznahorkay, in Handbook of Nuclear Chemistry, 2nd edition (Springer Verlag, 2011) 281-318

9. B. Biró et al., IEEE Trans. Nucl. Sci. 66, 1833 (2019). https://doi.org/10.1109/TNS.2019.2921102

10. K. Brezovcsik et al., Appl. Radiat. Isot. 160, 109127 (2020). https://doi.org/10.1016/j.apradiso.2020. 109127

11. S. Biri et al., Rev. Sci. Instrum. 83, 02 A341 (2012). https://doi.org/10.1063/1.3673006

12. Z. Juhász et al., Phys. Rev. A 82, 062903 (2010). https://doi.org/10.1103/PhysRevA.82.062903

13. R. Rácz et al., Plasma Source Sci. Technol. 26, 075011 (2017). https://doi.org/10.1088/1361-6595/aa758f

14. I. Lampé et al., Int. J. Nanomed. 14, 4709 (2019). https://doi.org/10.2147/IJN.S197782

15. S. Biri et al., High Energy Physics and Nuclear Physics - Chinese Edition 31, (2007) S165. http://caod. oriprobe.com/articles/32641029/Upgraded_TrapCAD_Code.htm

16. E. Naselli et al., Plasma Source Sci. Technol. 28, 085021 (2019). https://doi.org/10.1088/1361-6595/ ab32f 9

17. H. Koivisto et al., Nucl. Instrum. Methods B 94, 291 (1994). https://doi.org/10.1016/0168$583 \mathrm{X}(94) 95368-6$

18. R. Rácz et al., Proceedings of ECRIS 2014: the 21st International Workshop on ECR Ion Sources (Russian Academy of Sciences (RAN), Moscow, Russia 2014) p. 135

19. R. Rácz et al., Rev. Sci. Instrum. 83, $02 \mathrm{~A} 313$ (2012). https://doi.org/10.1063/1.3662960

20. www.highvolteng.com

21. www.atomki.hu/en/instruments/view/43

22. I. Rajta et al., Nucl. Instrum. Methods B 109(110), 148 (1996). https://doi.org/10.1016/0168583X(95)00897-7

23. www.pelletron.com

24. www.rayspec.co.uk

25. Gy. Gyürky et al., Phys. Rev. C 95, (2017) 035805. DOI: https://doi.org/10.1103/PhysRevC.95.035805

26. Gy. Gyürky et al., Phys. Rev. C 100, (2019) 015805. DOI: https://doi.org/10.1103/PhysRevC.100.015805

27. J. Gulyás et al., Nucl. Instrum. Methods A 808, 21 (2016). https://doi.org/10.1016/j.nima.2015.11.009

28. A.J. Krasznahorkay et al., Phys. Rev. Lett. 116, 042501 (2016). https://doi.org/10.1103/PhysRevLett. 116.042501

29. A.J. Krasznahorkay et al., Acta Phys. Pol., B 50, 675 (2019). https://doi.org/10.5506/APhysPolB.50.675

30. P. Hajdu et al., Vacuum 164, 15 (2019). https://doi.org/10.1016/j.vacuum.2019.02.046

31. Cs. Hegedűs et al., Materials 9, (2016) 25. https://doi.org/10.3390/ma9010025

32. P. Hajdu et al., Appl. Sci. 10, 7063 (2020). https://doi.org/10.3390/app10207063 